\title{
Vaccine Failure in Poultry Production and its Control Methods: A Review
}

\author{
Nigist Birhane $^{1}$ and Haben Fesseha ${ }^{2 *}$ \\ ${ }^{1}$ College of Veterinary Science, Mekelle University, Mekelle, Ethiopia \\ ${ }^{2}$ School of Veterinary Medicine, Wolaita Sodo University, Wolaita Sodo, Ethiopia \\ *Corresponding author: Haben Fesseha, School of Veterinary Medicine, Wolaita Sodo University, P 0. Box 138, Wolaita \\ Sodo, Ethiopia
}

\section{ARTICLE INFO}

Received: August 01, 2020

Published: 幽 August 18, 2020

Citation: Nigist B and Haben F. Vaccine Failure in Poultry Production and its Control Methods: A Review. Biomed J Sci \& Tech Res 29(4)-2020. BJSTR. MS.ID.004827.

Abbreviations: ND: Newcastle Disease; AI: Avian Influenza; IBD: Infectious Bursal Disease; IB: Infectious Bronchitis; DOI: Duration of Immunity; MHC: Major Histocompatibility Complex

\begin{abstract}
Vaccines and Vaccination programs may be considered as insurance against different infectious diseases. A successful vaccination program is dependent on many factors including vaccine handling, quality, and nature of the vaccine, use of local antigens, and immunogenic response inside the body of the bird and following the Manufacturer's instructions. The potential threat of disease outbreaks even in vaccinated flocks cannot be avoided completely, due to vaccine failures. Vaccine failure could be because of host factors such as stress, interference with maternally derived antibodies, lack of booster doses, and vaccination for immunosuppressed flocks, use of inappropriate dosage and route and untimely vaccination schedule. Moreover, it could also be due to antigen factors such as improper storage, use of expired vaccine and improper vaccine formulation. However, these factors can be avoided by proper vaccine storage, use of an adjuvant, none use of expired vaccines, use of stabilizers, practicing proper vaccination schedule, preparing flocks for vaccination, supplementation of vitamins and minerals and following vaccine manufacturer's guideline. Therefore, chicken farmers should follow stick biosecurity to prevent the outbreaks and vaccine manufacturers guidelines for storage, timing, and due dates, consult veterinarians before any vaccine administration and monitor the health status of chickens before vaccine administration.
\end{abstract}

Keywords: Antibody, Antigen; Control Measures; Immunization; Poultry, Risk Factors; Vaccine Failure

\section{Introduction}

Poultry production provides different types of animal proteins in the form of meat and eggs. The demand for chicken meat has increased over time because of the increasing demand for quality food in the form of meat and eggs. Poultry birds are prone to different types of fowl diseases and result in high economic losses to the poultry industry especially in developing countries $[1,2]$. Even though chickens are susceptible to bacterial, viral, parasitic and fungal infections, the outbreaks due to viral infection such as Newcastle disease (ND), Avian Influenza (AI), Infectious Bursal Disease (IBD), Infectious Bronchitis (IB), cause a great production loss in terms of reduction in meat and egg production and is the major constraints of the poultry sector [3,4]. In Africa and Asia with developing economies, poultry disease remains the greatest threat to the poultry industry and is responsible for very large economic losses to the producer [1]. The high prevalence of poultry disease creates major constraints in the development of the poultry sector, and this requires immunization intervention of chickens before disease entry $[5,6]$. Different strategies can be implemented to effectively prevent and control the entry and spread of poultry diseases at international, national and farm levels and poultry disease control plans often include the use of vaccination $[6,7]$.

In late 1800 , the first poultry vaccine was introduced and currently poultry vaccines are widely used for the prevention and control of most important infectious poultry diseases [8]. A vaccine 
is a biological preparation that are administered at the optimal and safe amount to enhances active acquired immunity to a particular disease. They are synthesized using several techniques such as eggbased vaccines, cell-based vaccines, and vaccines produced using investigational-manufacturing (plant, bacterial culture, and insect cell) systems. The immunization of birds is generally accepted as the most cost-effective and sustainable method of controlling infectious fowl diseases. This is mostly achieved by inoculation of non-pathogenic (attenuated or killed forms of the microbe containing its toxins or one of its surface proteins) that have great potential to elicit and boost the immunity of the host cell against diseases [9]. According to different research outputs, chickens are the most vaccinated farm animal species in recent days. For instance, a broiler chicken receives an average of eight different vaccines whereas a layer chicken may receive an average of around twelve different vaccines during their production cycles. Even though vaccines are used extensively in different poultry farms, it has also some limitations in control infectious poultry diseases using vaccination [8].

A vaccination failure arises when birds fail to develop adequate antibody titer levels and/or are at a risk of field disease outbreak. According to different research outputs, vaccination failure occurs due to inappropriate vaccines schedule (timing), improper handling of vaccine, vaccine quality, vaccine strain/serotype and insufficient amount of antibody titer levels after vaccination that predisposes the chickens to a field disease outbreak. Moreover, immunesuppression, maternal antibodies, stress, and management practices were also known causes of vaccination failure in poultry flocks $[10,11]$. Vaccines have a significant role in disease prevention and control worldwide. This, in turn, has a great importance in minimizing the emergence of outbreaks at the farm level and contributes a lot to the growth of poultry production. There different type of vaccines and vaccination schedule throughout the world and their efficiency depends on several factors such as the method of production, biosecurity level, disease pattern, maternal immunity, availability of vaccines, costs and potential losses [12-14]. Even though different methods have been applied and recommended for controlling infectious diseases in poultry, vaccination was found as the most important tool [4]. Thus, Immunization is the process of boosting immunity using biologically prepared antigen in the form of vaccines and used as prophylactic measures against specific pathogens [5].

The aim of vaccine is to immunize the birds through inoculation of non-pathogenic components of the pathogen in question, or closely related organisms that is nearly similar to naturally acquired immunity. This provides assurance to reduce the clinical and production impacts of the infections rather than the prevention of various infections outbreaks in poultry flocks $[10,15]$. Proper vaccination also used to develop a very high antibody levels in hens to increase the maternally derived antibodies (passive immunity) in the hatched offspring to protect the chicks against different infectious diseases during the early phase of life $[3,4]$. Vaccination can be performed using live non-attenuated or attenuated vaccines. Live attenuated vaccine is a type of vaccine that is contains attenuated viral strains whose virulence is reduced via series of cell culture or embryo passages that induces both cell- and antibodymediated immune responses by stimulating the body's immune response [16]. Newcastle disease, infectious bronchitis, infectious bursal disease, and other bacterial vaccines are commonly used live vaccines against diseases of poultry [17]. Unlike live attenuated vaccine strains, the non-attenuated vaccines have a great potential to cause disease in the vaccinated host especially in chickens with decreased immunity and are not preferred as a first choice [12]. This is due to reversion to virulence during virus replication in the host and inadequate attenuation results in clinical disease and adverse vaccine reactions and this causes great difficulty in controlling the disease $[4,6]$.

The use of a live attenuated vaccine strain has a great advantage since it can be administered through the natural route of infection and enables to induce mucosal and systemic immunity $[13,18]$. Moreover, it helps with a large number of flocks since it is easy and suitable for administration [12]. Inactivated or killed virus vaccine is considered safer than the conventional live attenuated vaccines since are they are less immunogenic and do not promote the endogenous production of antigenic proteins stimulating the cellmediated immune response [2]. Such vaccines are made inactive by using physical (use of ultraviolet radiations and heat) and chemical (use of formalin) means to inactivate their pathogenicity, but the protein coat structure has been maintained, which acts as immunogenic. Most poultry vaccines were either inactivated organisms that were formulated with an oil-based adjuvant or live attenuated vaccines and were not very effective in many cases. Thus, the discovery of antigen/gene delivery systems has facilitated the development of novel prophylactic and therapeutic veterinary vaccines. Recently, reverse vaccinology is the bestapproach and uses several bioinformatics algorithms to predict antigen localization and it has been successfully applied to immunize against many veterinary diseases [19].

There are several options for application of the immunization in poultry and these should fit to the conditions of the disease in the locality, the standard of farm biosecurity, and the level of challenge for each type of poultry operation. Inactivated or Killed vaccines confers a long-term immunity for the flocks [20]. So, successful vaccination with inactivated vaccines requires multiple inoculations and the presence of adjuvants to enhance immunogenicity $[3,13]$. Furthermore, as the inactivated vaccine does not replicate in the host after administration, it is generally considered unsuitable for mass application and needs to be administered by intramuscular injection [4]. During vaccination programs, the overall costbenefit analysis should be considered. These includes, costs 
associated with the service delivery that is cost of vaccine, labour, and equipment, nursing, laboratory testing, and all other related activities [21]. Besides, vaccination or failure to gain immunization even after vaccination is the most common complaint by producers and farmers on the basis of different previous research findings [7]. Accordingly, vaccine failure is caused due to lack of developing adequate immunization of birds after vaccination and exposes them to different infections [1].

In developing countries, the most prevailing problems related with high rates of vaccination failures in poultry flocks are improper transportation, handling, storage, and administration of vaccines $[2,6]$. Consequently, this paper was organized to enlighten about vaccine failure in poultry and its control measures.

\section{Risk Factors for Vaccine/ Immunization Failure}

Poultry vaccines are widely applied to prevent and control contagious poultry diseases. The effective application of vaccine and vaccination program requires good quality vaccines and proper application. There are several factors that cause immunization failure in vaccinated poultry and all these various factors should be considered with equal importance. In effect, failure to respect any of the following one will compromise the overall quality of the vaccination [6]. Among these exposure to overwhelming level of infectious agents, poor quality of vaccines, antigenic differences between the existing vaccine and circulating strains in the field instability in the cold chain, inappropriate application, and shortage of skilled veterinary technicians to administer the vaccine. Furthermore, the duration of immunity (DOI) after vaccination especially the length of time between vaccination and protection by vaccine and the shortage of DOI in animals under adverse conditions may not adequate. In addition, vaccination of stressed or fowls incubating disease causes sickness or death loss on the flock often blamed on vaccine [2,22].

\section{Problems Associated with the Vaccine}

Inactivation of the Vaccine Due to Expiration: The effectiveness of vaccine depends on the viability of the agents (virus and bacterial) in the vaccine and the replication in the birds. Thus, vaccine failure can be caused due to lack of proper handling (failure to properly store the vaccine at a suitable temperature) and improper administration of vaccines that may result in failure in immune response in apparently healthy birds. The potency of vaccines is maintained to a certain period; however, its viability may be lost due to past their expiration date even it is stored under the appropriate temperature. Moreover, the vaccine can be inactivated if it is mixed with different types of residual disinfectant on the syringes and needles $[13,23]$.

Antigenic differences between Existing Vaccine and Field Strains: Lack of vaccine efficacy due to antigenic variation amongst the vaccine and field strains (such as antigenic drift, antigenic shift) are usually a problem with killed vaccines than modified live vaccines. Accordingly, effective antibody-mediated immunity is developed if the antibodies exactly bind to the specific antigenic strain on the bacterial or viral surface. In contrast to antibodymediated immunity, cell-mediated immunity lacks strain specific immunization. Thus, it is necessary to isolate the circulating field strain and compare it with the existing vaccine strain $[3,21]$.

Inadequate Level of protection: The vaccine strain should elicit effective and sufficient immune response, and this depends of the antigenicity of viral strain. Eggs are the source of maternal antibodies or passive immunity in poultry and remains effective for the first two weeks. After proper vaccination, most poultry vaccines reach their peak response typically between 2-6 weeks and the protection or immunity level then starts to gradually decline. If birds are typically vaccinated with virulent infectious agents during these periods of time where protection by maternal antibodies reaches high, the vaccine may fail to stimulate immune response due to neutralization $[4,8]$. Even though birds are frequently vaccinated, the bird may become susceptible to different poultry diseases before they develop their own active immune responses. It is advised to give booster dose on the recommended schedule to develop the better protection level $[6,24]$.

\section{Factors associated with the Host/Birds}

After proper vaccination, the host requires few days to develop an effective immune response. If the birds infected by an infectious agent before and during the time of vaccination, the vaccine fails to produce sufficient antibodies resulting in apparent vaccination failure. Moreover, the following limitations are considered as the common cause for vaccine failure [7].

Stress Factors: Stress is a non-specific response to change in the environment that poses an excessive demand on the physiological and behavioral adaption abilities of the birds. The body composition of birds normally has inadequate resources to defend itself from diseases and environmental changes. Different predisposing factors cause stress in poultry birds. This includes stress due to poor ventilation, cold, heat, excess humidity, transportation, high stocking density (overcrowding), low spacing, improper sanitation, excess load of litter, under and malnourishment (nutritional deficiency), parasitism, fever, and so on [4, 23]. Since poultry birds are very much sensitive to extreme weather conditions, health-related and other management problems and this, in turn, lead to reduced immune response. Accordingly, chickens become generally unresponsive to vaccinations and are susceptible to infection during disease outbreaks. Furthermore, live vaccines can cause huge outbreaks if it is administered to immunocompromised chickens. Hence, stress predisposes chicken to reduced immunity, vaccine failure and economic losses due to low production [1].

Interference with Maternal Antibodies/ Immunity: Poultry are regularly vaccinated against different infectious diseases on 
the basis of their disease prevalence and the newly hatched chicks are passively immunized due to maternal antibodies in their blood. According to some research findings, maternal immunity can protect the chickens from various infectious diseases such as Newcastle disease virus and Gumboro (IBD) disease during the first week of birth. However, these maternal antibodies have potential to interact with the vaccine antigens and result in neutralizing antigen. Thus, vaccines such as live vaccines affect the development of immunity production by reducing the level of antibodies in the newly hatched chicken if they are vaccinated for first weeks $[13,25]$.

\section{Immunosuppressive and Coexisting Diseases:} Immunosuppression is caused due to compromised immune response and resulted in increased risk of infectious diseases in flocks of birds such as mycotoxicosis, infectious bursal diseases (Gumboro), infectious anemia, Marek's disease, and others. All this stress conditions resulted in impaired immune response that may also lead to vaccine failure and affect effective immunization $[6,13]$. This may result in the development of limited protection from vaccination and an excessive vaccine reaction including morbidity and mortality [26]. Vaccines are expected to vigorously stimulate the immune system. Thus, the presence of any concurrent mild local and systemic diseases may result in reduced vaccine efficacy. Additionally, the effectiveness of vaccine will be reduced if the infected or stressed birds are vaccinated for the same disease since the vaccine antigens are neutralized due to reaction with naturally produced antibodies against infectious pathogens. This causes vaccine reaction in birds and the disease condition may worsen and leads increased morbidity and fatality rate. Accordingly, it is highly recommended and important to secure the health status of birds before vaccination $[2,4,6]$.

Genetic Factor: Response to vaccine varies depends on the type of species of birds. The structure of major histocompatibility complex (MHC) are responsible for the individual variation of birds and this helps to determine the response of birds towards the bacterial and viral antigens. Birds might be more susceptible to pathogen due to the lack some structure in MHC helps to recognize one of the antigens [21,27].

\section{Factors associated with administration of vaccines}

Lack of proper Storage and Vaccine Instability in Cold Chain: Vaccines should be properly stored, transported, and applied as per the manufacturer's recommendations. In developing countries, the common encountered problems during vaccine storage that led to inactivation of vaccine are shortage of storage equipment, inappropriate storage temperature, lack of functional and effective refrigerators, mixing vaccines with other food items, interaction of vaccine with the disinfecting agents [23]. According to Nelson et al., [28] trying to store or transport the vaccine without proper preservation, handling and following cold chain results in denatured antigen thereby ends with vaccine failure. Antigens are killed when the vaccine is exposed to direct sunlight which leads to reduced antigen concentration in the vaccine and vaccine effectiveness. Therefore, the vaccine should be well placed and protected from direct sunlight for better efficiency $[2,6]$.

Instability of vaccines regularly occursand are the main challenge during clinical development and commercial distribution. There are different sources and mechanisms of vaccine instability including the complex and delicate nature of antigen structures (viruses, proteins, carbohydrates, protein-carbohydrate conjugates), the use of adjuvants to further boost immune responses, development of physicochemical and biological assays to evaluate vaccine integrity and potency, and the stabilization approaches to protect vaccine antigens and adjuvants as well as their interactions during storage [29].

Inappropriate Route of Administration: Poultry vaccines can be administered through oral, subcutaneous, intramuscular, wing web, drinking water, eye dropping, and spray. If a vaccine is not delivered using appropriate vaccination site or recommended route of administration, it may result in poor protection by the vaccine in poultry flocks. Besides, mass vaccination through drinking water and spray (aerosol) lacks uniformity in dosage between individual birds during administration that results in lack of effective immunity $[2,6]$.

Insufficient Dosage: The use of incorrect dosage such as under and overdosage of vaccines results in vaccine failure and vaccine reaction [4]. There are various factors that contributes for improper formulation of vaccine dosage. Among these, the high level of chlorine in water during vaccine preparation, use of water having antimicrobial contents, and miscalculation of vaccine dosage. Additionally, the use of vaccine for large flocks of bird's application beyond the recommended dosage of the manufacturer, it will be resulted in inadequate dosage that causes low level of vaccine titers and inactivation of vaccine [17].

Improper Formulation of Vaccine and Diluent used: Poultry vaccines should be prepared and properly formulated using specific diluent (saline water) as per the manufacturer's guidelines. Some diluents are specific for a single vaccine (diluents for lyophilized vaccines) and contains some preservatives that can demolish the activity of the other vaccine. Improper dilution and lack standard procedure of vaccines formulation using the diluent in a single syringe may also decrease the potency of vaccines. Thus, during formulating different type of vaccines in a single syringe, the possible outcome after interaction should be adequately examined $[6,30]$.

Use of Single Adjuvants: The use of single adjuvant has several drawbacks since it does not fulfill the requirement of all the vaccine requirements. Induction of weak, inadequate, and short- 
lived immune responses are among the limitations of using single adjuvant [31].

\section{Managemental, Technical and practical Errors}

Hygienic Practices : The vaccine can increase the animal's resistance to disease, but this resistance can be overwhelmed if good management practices are not followed. Poor sanitation together with other factors such as stress, overcrowding, concurrent infection reduced the effectiveness of poultry vaccines. Without clean-out and disinfection over successive flocks, the challenge dose might be too high, or infection might occur too soon [8,27].

Failure due to Concurrent Administration of Multiple Vaccines: Poultry vaccines are commonly administered concurrently to birds for various type of infections. However, there are limited literatures that describes about the safety and efficacy of when administering multiple vaccines. According to different studies, in some vaccines, there is a possibility of reduced the antibody response to the vaccine when administered in combination and increase the risk of morbidity and mortality [22,27].

Faulty Timing and Schedule of Vaccination: Mostly the recommended time vaccination in poultry is during cold hours of the day especially early in the morning and late hours of the day or evening. This is the most suitable and comfortable time of vaccination to get a better response from the vaccination. However, the outcome of the vaccine may be less in vaccinated flocks if the birds are vaccinated during the hot hours of the day (heat stress) $[4,10]$. During the time of vaccination, it is important to consider the age of the bird. Some antigenic receptors are age specific since the develop starting from day old (as early as they hatch of a chick) to old age. In early age, birds start to develop the receptors of Newcastle disease, infectious bronchitis, and infectious bursal disease (IBD) whereas the receptors for infectious bursa disease and fowl pox develop with advancing age. Accordingly, it is advisable

Table 1: Summary of the Factors that deteriorates vaccine efficacy.

\begin{tabular}{|c|c|}
\hline Factor & Description \\
\hline Maternal immunity & Earlier interfere with antigens of the vaccine \\
\hline Concurrent disease with immunosuppressive agents & $\begin{array}{l}\text { Birds immune system fail to produce sufficient immune response against } \\
\text { the infectious agents }\end{array}$ \\
\hline Mismatch between field virus and the vaccine (Pathotype of field strain) & $\begin{array}{l}\text { Lack of antibodies similarity between the circulating viral strain (field } \\
\text { virus) and the vaccine strain to produce full protection }\end{array}$ \\
\hline The quantity of Antigen and immunogenicity of the vaccine & $\begin{array}{l}\text { An adequate immune response is not induced if a load of antigen is not } \\
\text { enough to elicit immune response. }\end{array}$ \\
\hline Health status of the chickens & When the bird's immune system does not produce a sufficient response \\
\hline Vaccine coverage (the total number of birds vaccinated) & Large number of birds must be protected to restrict the transmission \\
\hline Species or breed of bird & $\begin{array}{c}\text { During preparation, the target species should be considered for optimal } \\
\text { response }\end{array}$ \\
\hline Duration of immunity or & $\begin{array}{l}\text { Immunocompromised birds are exposed to infection and disease since the } \\
\text { immunity declines }\end{array}$ \\
\hline Quantity of doses & Booster doses should be given to improve immunity over time \\
\hline
\end{tabular}

to vaccinate birds after development of this receptors in the bodies since lack of receptors for the antigen in the host result in vaccine failure $[4,13]$.

Usage of Local Antigens or Serotype: Infectious poultry diseases such as Gumboro (IBD) and salmonellosis have different serotypes. Besides, there is variation in the prevalence of the serotypes differ from area to area. Identification of locally circulating infectious agents in different areas are most important for vaccine production. Thus, these locally isolated antigens (immunogens) are used for manufacturing of vaccines. Disease outbreaks can be occurred in the area if these local antigens are not used during production [6]. Most imported vaccines may contain serotypes that differ from the circulating local or field strain and may not be effective to tackle high virulent strains of different nature $[7,8,24]$.

Lack of Repeat or Booster Dosage: A booster dose is a dose of immunization dose. Thus, administration of repeated dose in fixed period of time depending on the type of vaccine. Besides, earlier immunization is necessary for introduction of the antigen of the vaccine into the birds' body whereas booster dosage is mandatory to maintain maximum level of protection against an antigen. However, low level of antibody titers is caused due to the absence of booster dose that in turn results in failure of vaccine [4].

Lack of Strict Biosecurity: Biosecurity is a compulsory comprehensive practice in poultry farms to protect the farms both from intentional and unintentional biological threats. Thus, the lack of inclusive biosecurity strategies makes susceptible to different poultry disease outbreaks. This makes the chicken immunocompromised and contributes to vaccine failure as a result of the change of the vaccine strain to pathogenic one [22]. Accordingly, numerous practical aspects affect vaccination and contribute to vaccine failure (Table 1 ). antigen designed to develop effective immunization after an initial 


\begin{tabular}{|c|c|}
\hline Quality of administration & $\begin{array}{c}\text { An optimal and full dose should be administered properly with proper } \\
\text { equipment. }\end{array}$ \\
\hline $\begin{array}{c}\text { The Time or Speed to develop optimum level of protection after } \\
\text { vaccination }\end{array}$ & $\begin{array}{l}\text { Any exposure before sufficient development of sufficient immunity may } \\
\text { lead to infection }\end{array}$ \\
\hline
\end{tabular}

Note: Source: [22].

\section{Mitigation Strategies for Vaccine Failure}

Poultry vaccines are commonly used in several types of production systems. A cost-benefit assessment should be conducted to identify the suitable approach available before implementing the control options, while considering various situations [8]. For instance, applying stamping out measures to control major poultry diseases poses a great impact on the long-term sustainability of farm production and on the livelihood of smallholder farmers in developing countries, where there are limited compensation measures [13]. Thus, cost-benefit analysis should consider the following conditions such as: the species of birds, the production system, fowl densities, the antigenicity/virulence of the viral strain involved, provision of veterinary facilities, and the impact on trade $[4,12,27]$. The following strategies are considered as a best option to control the major infectious and contagious poultry diseases.

\section{Proper Formulation of the Vaccines}

It is always recommended to follow the instruction provided by the vaccine producer since there are some variation in preparation and formulation of the vaccines. For instance, the viable components of vaccines can be destroyed during preparation if the chemicals (sanitizers) used to clean the drinkers are mixed with the vaccine. In addition, during preparation of vaccines, the timing, the duration of efficacy, and the type of diluent should be considered. Furthermore, any remained part of the vaccine after vaccine preparation should be discarded carefully in the appropriate place. The duration of efficacy must be considered since vaccines against such as IBD and pox diseases lose its $100 \%$ and $50 \%$ of their potency after 1 hours of their reconstitution, respectively [10].

\section{Attention to Maternal Immunity}

In most poultry farms, fowls especially parent stocks are regularly vaccinated depending on the various outbreaks and prevalent infectious disease of birds. Thus, newly hatched day-old chicken by default has developed passive immunity in their blood from their parent stock through antibodies transferred in eggs. however, when vaccines especially live one administered during the first two weeks where these maternal antibodies are reaches their peak, the vaccine will be neutralized. According to different research findings and poultry manuals, vaccine should be given for newly hatched chicken based on the antibody titration level against the proposed vaccines to get the maximum immunization from the vaccine. For instance, the recommended time of vaccination for Gumboro disease (IBD) should have a gap of minimum of 11 days post hatching whereas for Newcastle disease, the chicken should be 7 days old at the time of vaccination [32].

\section{Proper Storage and Maintain Cold Chain Temperature}

Poultry vaccines should be properly stored and transported at the optimum temperature as per the manufacturer's standard since temperature variation has a direct effect on the efficacy of the vaccine $[4,21]$. Besides, during storage the vaccine must be stored separately from other items such as food item, chemical, pathological samples. For this reason, certain live vaccines like Marek's disease vaccines are easily inactivated due to improper handled and storage $[13,23]$. The proper cold chain temperature should be maintained to keep the viability of the vaccine. However, there are several factors that affect the cold chain temperature like shortage of electric source and lack of cooling systems (refrigerator), over chilling, and lack of vaccine transporting equipment (ice box). Likewise, the extra chilling of oil-based vaccines results in reduced potency of vaccines due to crystal formation of adjuvants (aluminum salts) in the vaccines [13]. Thermostable vaccines are produced in such a way to cope up with the fluctuation of temperature such as cold and hot environments, while freeze-dried vaccines should be stored in the refrigerator at $4{ }^{\circ} \mathrm{C}$ and transported using ice blocks/cooling system to keep at the recommended temperatures $[13,23]$. The use of thermostable vaccines can be an alternative to overcome the difficulties related to cold chain and storage temperature [23,33].

\section{Avoiding Use of Expired Vaccines}

Most poultry vaccines that are produced worldwide have different table shelf life and is clearly described on the leaflets prepared by manufacturer. For instance, some vaccines like live lyophilized vaccines, have wide range (1-2 years) of shelf life whereas other like oil-based vaccines have limited range (3-6 months) of shelf-life [23]. The vaccine vial should be carefully checked and assessed for the date of expiry prior to application. The use of expired vaccines must be avoided and advisable to discard them properly or replaced with the new one from the manufacturers $[13,21,23]$.

\section{Use of Adjuvant and Stabilizers}

An adjuvant is a supplementary substance that are added to the vaccine to increase the bioavailability of vaccines and to enhance immune response to the target antigen [34]. This in turn, helps to increase the immunogenicity of vaccines to confer long-term protection and to reduce the required antigenic dose to produce effective immunity [31]. Adjuvants have a potential effect on 
inducing a mucosal immune response that help to improve the safety of the vaccine. For instance, vaccines given orally faces a great difficulty due to the microbial barriers in gastrointestinal tract and the antigen must be protected using adjuvants to overcome this problem that helps to activate the immune response [1334]. A combination adjuvant platform is promising and beneficial for suboptimal vaccines and particularly advantageous for vaccines against specific and more susceptible flocks of poultry populations. In contrast single adjuvants, combined adjuvants act synergistically by stimulating and activating various types of immune cells such as dendritic cells, macrophages, lymphocytes, etc [31].

A stabilizer is a substance that is added to a vaccine in order to stabilize and increase the shelf life of the vaccine during formulation and administration of different vaccine. There are different types of stabilizers available in the market for the various kind of vaccine preparation. For example, for live oral vaccines such as infectious bronchitis, Newcastle disease, Gumboro disease, Vac-Safe (Intervet) and Vital Blue are the best stabilizers. Besides, locally available materials like skimmed milk at the rate of $2 \mathrm{~g} / \mathrm{L}$ can also be added to vaccine and used as substitute for other stabilizers [21,32].

\section{Controlling Stress and Use of Antimicrobials}

Stress can be managed by identifying the potential sources and causes that can be categorized into predictable stress that are related with management and handling problems and unpredictable stress related with variation in temperature, and different incidence of disease outbreaks. A method of assessing and identifying stress should be developed to control the vaccine failure due to unforeseen stress. For this reason, firstly, the feeding, housing, microbial flora, and breeding system should be adjusted in the proper standard to increase the quality of life and increase the immune response sources. Secondly, administration of different kind of therapies to minimize the level of immunosuppression in the birds due to non-specific or unidentified factors. However, such therapies should not be necessarily considered as the only means of prevent stress and its consequences. Besides, corrective measures should be taken after identifying the source of the stress such as reducing the bird density, increasing the number of feeders and drinkers, improving the ventilation and others $[8,13]$.

In poultry production, Antimicrobial can be used pre and post vaccination and are given to minimize and prevent the development of a high risk of secondary bacterial infections due to opportunistic microorganisms during the periods of stress. When selecting the antimicrobials compatible with the immune defense system, spectrum of activity, and its potential against the pathogenic opportunistic microbes. For instance, antimicrobial like Erythromycin have a special immunomodulating capacity through stimulating the secretion of interleukins by leukocytes that help to maintain strong immunity for the birds $[27,35]$. However, the vaccines should not be mixed with the antimicrobials. Thus, there should be sufficient duration of time for the antimicrobials and the antimicrobial should be administered before minimum of two days before and after administering the vaccination $[4,8]$.

\section{Administration of Vitamin and Mineral}

Vitamins and minerals should be provided to immunocompromised birds that supports the immune response by acting on the immune cell and rapidly enhances the production of antibodies; as a result, the birds get a better immunity in a shorter time. Administration of vitamins (vitamins A, E, C and B) in poultry feed helps to improve some of the adverse effects due to stress that help to support the immune defense system. Besides, the use of vitamins prior to a predictable stressing condition is very important and should remain till the whole stressing scenario ends. This should be done administered 24 hours prior to vaccination and vitamins are involved in all kinds of stress reaction and are used up directly during synthesis of glucocorticosteroids (Vitamin C) or indirectly by increasing the number in most intermediate metabolic reaction (Vitamin B). [13,36]. According to studies, the supplementation of additional vitamin and mineral is necessary to maximize the resistance to disease since routine poultry feed may not be sufficient to fulfil the demand of normal immunity $[37,38]$. The combination of vitamins (A, D3, E, B-group water-soluble vitamins), minerals (selenium) and amino acids have a great role in immune modulation and stimulating humoral and cellular immunity and also most effective mechanism to tackle vaccine $[18,39]$.

\section{Correct Vaccination Schedule and Strick Biosecurity}

A proper vaccination program should be there for poultry flocks depending on the prevalence of the disease in the area in order to minimize disease outbreaks and financial loss associated with the disease. it is better to vaccinate the birds prior to the incidence of the disease so that the birds develop the required amount of antibody titration. For instance, vaccination against infectious bronchitis and avian influenza should be given before winter period since the outbreak of diseases are commonly occurs in birds during winter [18,21]. Besides, biosecurity is the most important and crucial component of any poultry outbreak prevention and control strategy and should be maintained at a high level to complement vaccine use. In addition, it is advised to follow strict stamping-out (all in all-out) principles to avoid cross-contamination between flocks $[4,22]$.

\section{Flocks Preparation for Vaccination}

During administration of vaccines, the flocks of birds should be properly prepared for vaccination depend on the route of administration, age of the birds, technique of vaccination and type of vaccine. Besides, precautionary measures such withholding feed and water for 2-3 hours prior to vaccination, should be followed strictly to increase the efficacy of the vaccine. For instance, spray 
vaccination is administered today old chicks post-hatch in cabinets for mass-vaccination through aerosol method. While eye drop day, old chicks are applied to individual chickens through eye. Oral vaccination are the most common means of vaccine administration. The vaccination should be given after fasting the flock for few that later helps to finish the vaccine within short period (up to 2 hour) of time and the vaccines should be prepared and administered in clean equipment (drinkers). For this purpose, sufficient number of drinkers must be available and proper follow up as well as regular moving of birds ensure all birds drink sufficient amount of the vaccine $[4,21]$.

\section{Strictly follow the Manufacturer's Guidelines}

When applying vaccines, the type (inactivated, live attenuated, DNA and recombinant vaccines) and use of vaccine should be considered as per the guidelines put by the manufacturer like preparation, usage, storage, and route of administration. Hence, it is always advisable for vaccination to be given by trained or qualified veterinarian in order to minimized the adverse reactions related with the vaccine such as local tissue reaction (in activated or killed vaccines) and vaccine reaction (live attenuated vaccines). Moreover, most manufacturers advised to avoid any stressing environment (transportation, overcrowding and hot weather) by testing for proper titration level of antibodies before application of poultry vaccines. For instance, early or late hours of the day are considered as the most suitable periods of vaccination especially during summer $[2,10]$.

\section{Conclusion and Recommendations}

Infectious Poultry diseases are threat to the poultry producers and industry that results in great financial losses. Poultry vaccines have been used for several years and play an important role in promoting poultry health and welfare as well as increasing production. Vaccination is one of the most important tools and cost-effective method to protect fowls from disease and reduces a great loss as well as reduce the need for antibiotics in poultry products. However, there are challenges concerning poultry vaccines that contribute to vaccine failure including the instability of the vaccine, incorrect handling, preservation, and transportation as well as the administration that require new solutions in vaccine development. Hence, to address these problems, it is advisable to follow the vaccine manufacturer's guidelines that have a paramount importance controlling failure. Besides, when administering vaccine, the health of flocks and the level of maternally derived titers should be a consideration. Therefore, veterinarians working at different field clinics should follow vaccine manufacturers' guidelines for storage, timing, due dates, and administration. Also, chicken farmers and managers should consult veterinarians before vaccine administration, and also, they should monitor the health status of chickens before vaccination.
The goal of vaccination needs to be clearly defined and the farmer must take all the necessary steps to control both the practices used during the vaccination operation and external factors. Furthermore, vaccination helps to enhances the capacity to control the poultry diseases and should be implemented along with other disease control and eradication measures.

\section{References}

1. Abdullahi U, Adamu S, Ahmed A (2009) Investigating on some causes of poultry vaccination failure in Bauchi Metropolis and environs, Nigeria. Nigerian Journal of Experimental and Applied Biology 14(1): 47-50.

2. Bosha J, Nongo $\mathrm{N}$ (2012) Investigating vaccine handling and administration in Makurdi metropolis, Benue state, Nigeria. Paper presented at: Proceedings of $37^{\text {th }}$ conference of Nigerian society of animal production (NSAP), University of Agriculture Makurdi, Bennue state, Nigeria.

3. Terfa Z, Garikipati S, Dessie T, P Wigley, JM Bettridge, et al. (2015) Farmers' willingness to pay for a village poultry vaccine service in Ethiopia: Prospect for enhancing rural livelihoods. Food Security 7(4): 905-917.

4. Sharif A, Ahmad T (2018) Preventing Vaccine Failure in Poultry Flocks. Immunization-Vaccine Adjuvant Delivery System and Strategies: Intech Open.

5. Ramirez LA, Arango T, Boyer J (2013) Therapeutic and prophylactic DNA vaccines for HIV-1. Expert opinion on biological therapy 13(4): 563-573.

6. Bosha J (2012) Common breaches in poultry vaccine handling and administration in Makurdi Metropolis: A recurrent phenomenon in the tropics. Vom Journal of Veterinary Science 9(1): 11-16.

7. Nongo N, Bosh J (2004) Poultry Vaccine Handling and administration in Makurdi. A preliminary investigation. Proceeding of the Nigeria Veterinary Medical Association held at NVRI, Vom $20^{\text {th }}-24^{\text {th }}$ November.

8. Abdul Careem MF (2015) Main challenges in poultry farming: Vaccination failure. Zaragoza, Spain: Grupo Asis.

9. Smith D (2007) Understanding Vaccines: Institute of agriculture and Natural Resources, Neb Guide G, University of Nebraska-Lincoln Extension.

10. Butcher GD, Yegani M (2009) Investigating Vaccination Failure in Poultry Flocks. EDIS. 2009(1)

11. Müller H, Mundt E, Eterradossi N, Islam MR (2012) Current status of vaccines against infectious bursal disease. Avian Pathology 41(2): 133139.

12. Alexander DJ, Senne D (2003) Newcastle disease. Diseases of poultry 11(1): 64-87.

13. Hassan AM, Garba MS, Durodola OS (2017) Causes of Vaccination Failures Among Poultry Farms In Kano Metropolis, Kano State, Nigeria. Fudma-Journal of Agric \& Agric Tech 3(1): 104-110.

14. Ifrah M, Perelman B, Finger A, Uni Z (2017) The role of the bursa of Fabricius in the immune response to vaccinal antigens and the development of immune tolerance in chicks (Gallus domesticus) vaccinated at a very young age. Poultry science 96(1): 51-57.

15. Dana S, Rathore B, Kaul P (2000) Morbidity, and mortality pattern in desi chicken reared by the santal tribe of West Bengal. Indian Journal of Animal Research 34(1): 49-51.

16. Ganguly S, Paul I, Mukhopadhayay S (2010) Different types of vaccines and vaccination - The most accepted trend to control and eradicate infections. Indian Pet Journal 5: 34-37.

17. Wambura PN, Kataga S (2011) Putative protective antibody response following oral vaccination of multi-age free-ranging helmeted guinea 
fowls (Numida meleagris) with Newcastle disease virus strain I-2 coated on oiled rice. Tropical animal health and production 43(1): 99-102.

18. Sanda M (2015) Effects of Vitamin-Mineral Supplement on the Immune Response of Broilers to Newcastle Disease Vaccination. International Journal of Agricultural and Veterinary Sciences (IJAVS) 1(1): 10-13.

19. Jorge S, Dellagostin OA (2017) The development of veterinary vaccines: a review of traditional methods and modern biotechnology approaches. Biotechnology Research and Innovation 1(1): 6-13.

20. Furuya Y, Regner M, Lobigs M, Koskinen A, Müllbacher A, et al. (2010) Effect of inactivation method on the cross-protective immunity induced by whole 'killed' influenza A viruses and commercial vaccine preparations. Journal of General Virology 91(6): 1450-1460.

21. Marangon S, Busani L (2007) The use of vaccination in poultry production. Revue Scientifique et Technique-Office International des Epizooties 26(1): 265-274.

22. Spackman E, Pantin Jackwood MJ (2014) Practical aspects of vaccination of poultry against avian influenza virus. The Veterinary Journal 202(3): 408-415.

23. Evans M, Pope M (1995) Vaccine handling and storage in genera practice. Health trends 27(4): 124-126.

24. Aliu Y (2004) Responsible veterinary drug use: Vaccines and vaccination failure. Paper presented at: Continuing Education Paper on Emerging trends in Veterinary Practice in Nigeria, Merit House, Abuja.

25. Pitcovski J, Gutter B, Gallili G, Martin Goldway, Beny Perelman, et al (2003) Development and large-scale use of recombinant VP2 vaccine for the prevention of infectious bursal disease of chickens. Vaccine 21(32): 4736-4743.

26. De Herdt P, Jagt E, Paul G, S Van Colen, R Renard, et al. (2005) Evaluation of the enzyme-linked immunosorbent assay for the detection of antibodies against infectious bursal disease virus (IBDV) and the estimation of the optimal age for IBDV vaccination in broilers. Avian pathology 34(6): 501-504.

27. Shane SM (2005) Handbook on Poultry Diseases. (2 ${ }^{\text {nd }}$ Edn.). American Soybean Association, Singapore.

ISSN: 2574-1241

DOI: $10.26717 / B J S T R .2020 .29 .004827$

Haben Fesseha. Biomed J Sci \& Tech Res

CC) This work is licensed under Creative

Submission Link: https://biomedres.us/submit-manuscript.php
28. Nelson CM, Wibisono H, Purwanto H, Mansyur I, Moniaga V, et al. (2004) Hepatitis B vaccine freezing in the Indonesian cold chain: evidence and solutions. Bulletin of the World Health Organization 82(2): 99-105.

29. Kumru OS, Joshi SB, Smith DE, Russell Middaugh C, Prusik T, et al. (2014) Vaccine instability in the cold chain: Mechanisms, analysis, and formulation strategies. Biologicals. 42(5): 237-259.

30. Brugh Jr M, Siegel H (1978) Inactivated Newcastle disease vaccines: influence of virus concentration on the primary immune response. Poultry science 57(4): 892-896.

31. Garg R, Babiuk L, Gerdts V (2017) A novel combination adjuvant platform for human and animal vaccines. Vaccine 35(35): 4486-4489.

32. Nwanta J, Umoh J, Abdu P, Ajogi I, Egege S (2005) Comparison of the Cost of Unvaccinated and oral Vaccinated Local Chickens with a Malaysian Thermostable Newcastle Disease Vaccine (NDV4HR) In Kaduna State, Nigeria. Bulletin of Animal Health and Production in Africa 53(3): 203210.

33. Alders R, Spradbrow P (2011) SADC planning workshop on Newcastle Disease control in village chickens. Paper presented at: Southern African Development Community planning workshop on Newcastle Disease control, Maputo, Mozambique, 6-9 March 2000.

34. Lavelle EC, O'Hagan D (2006) Delivery systems and adjuvants for oral vaccines. Expert opinion on drug delivery 3(6): 747-762.

35. CEVA (2005) Factors affecting vaccination. Vaccines and Vaccination in Poultry Production: Santé Animale pp. 81-98.

36. Gershwin M, Beach R, Hurley L (1985) The potent impact of nutritional factors on immune response. Nutrition and Immunity. Academic Press, New York, USA, p. 1-7.

37. Lin YF, Chang SJ (2006) Effect of dietary vitamin E on growth performance and immune response of breeder chickens. Asian-Australasian journal of animal sciences 19(6): 884-891.

38. Nockels C, Odde K, Craig A (1996) Vitamin E supplementation and stress affect tissue $\alpha$-tocopherol content of beef heifers. Journal of animal science 74(3): 672-677.

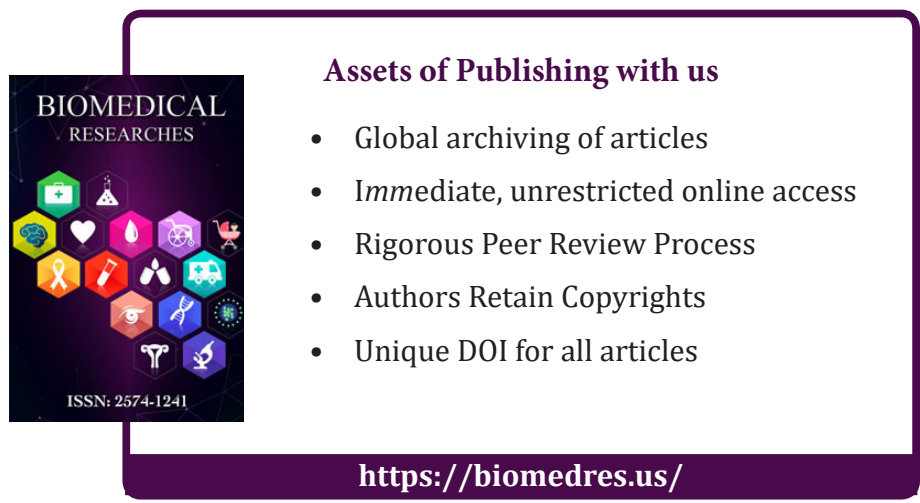

Copyright@ Haben Fesseha | Biomed J Sci \& Tech Res | BJSTR. MS.ID.004827. 